\title{
Baudouin de Sebourg, publié par Larry S. Crist
}

\section{Anna Maria Finoli}

\section{(2) OpenEdition}

\section{Journals}

\section{Edizione digitale}

URL: http://journals.openedition.org/studifrancesi/32762

DOI: 10.4000/studifrancesi.32762

ISSN: 2427-5856

\section{Editore}

Rosenberg \& Sellier

\section{Edizione cartacea}

Data di pubblicazione: 1 décembre 2005

Paginazione: 609-611

ISSN: 0039-2944

\section{Notizia bibliografica digitale}

Anna Maria Finoli, «Baudouin de Sebourg, publié par Larry S. Crist», Studi Francesi [Online], 147 (XLX I

III) | 2005, online dal 30 novembre 2015, consultato il 20 avril 2021. URL: http://

journals.openedition.org/studifrancesi/32762 ; DOI: https://doi.org/10.4000/studifrancesi.32762

\section{Questo documento è stato generato automaticamente il 20 avril 2021.}

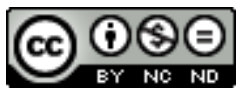

Studi Francesi è distribuita con Licenza Creative Commons Attribuzione - Non commerciale - Non opere derivate 4.0 Internazionale. 


\title{
Baudouin de Sebourg, publié par Larry S. Crist
}

\author{
Anna Maria Finoli
}

\section{NOTIZIA}

Baudouin de Sebourg, publié par Larry S. CRIST, Paris, Société des Anciens Textes Français, 2002, voll. 2, pp. CIII 1229.

1 Il Baudouin de Sebourg non può essere considerato una vera e propria chanson de croisade, ma ha certo molti rapporti con il secondo ciclo della Crociata. Il vasto poema (più di 25.000 alessandrini) si apre con la genealogia di Baudouin, futuro re di Gerusalemme, figlio di Ernoul de Beauvais, fratello di uno dei Chetifs, e di Rose, sorella di Elyas e degli altri enfants-cygne, narra quindi le enfances Baudouin, e poi le altre imprese del protagonista, inserendole in una trama fittissima di avvenimenti che coinvolgono la sua famiglia, numerose donne, anche di origine saracena, fratelli, cugini, figli, tra cui il Bâtard de Bouillon, eroe di un'altra canzone di gesta.

2 Il Crist si avvale della collaborazione di R.F. Cook, già coautore del volume Le deuxième cycle de la Croisade. Deux études sur son développement. Les textes en vers (Genève, Droz, PReF, CXX, 1972), e autore, tra l'altro, dell'edizione critica del Bâtard de Bouillon (Genève, Droz, TLF, 187, 1972) conservata in uno dei due mss. della Bibliothèque Nationale de France, (A, f.fr. 12252, e B, f.fr. 12553) che hanno tradito il Baudouin de Sebourg. Il Cook che, come il Crist, ha acquisito una notevole competenza per quanto riguarda la letteratura epica sulle Crociate, ha redatto l'Introduzione e le note al testo del ms. A e preparato il glossario servendosi di concordanze elettroniche precedentemente elaborate.

3 L'introduzione costituisce un'esposizione sintetica, con gli opportuni rinvii agli studi particolari, dei vari problemi che l'opera pone. Il primo capitolo è dedicato alla data di composizione (metà del XIV secolo), alla patria (regione di Valenciennes) e alla personalità dell'anonimo autore, argomento quest'ultimo controverso, che ha indotto 
qualche critico a formulare l'ipotesi di due o più autori. Segue l'analisi della lingua del testo e dello scriba, della struttura e della composizione della chanson de geste, la rassegna dei rapporti con i testi del secondo ciclo della crociata e con altre opere della tradizione letteraria; un notevole spazio è infine dato all'esame della versificazione, con l'ausilio anche di tabelle numeriche. Completano l'edizione, com'è consuetudine, prima del testo il riassunto degli avvenimenti narrati, e alla fine le note, l'indice dei nomi propri, la lista dei proverbi corredata da un indice delle parole-chiave, un glossario, la bibliografia degli autori citati, la tavola delle corrispondenze con la precedente edizione Boca.

4 Il testo, tradito come si è detto da due mss, uno dei quali (A) è di poco posteriore alla creazione dell'opera, era già stato pubblicato da L.-N. Boca nel 1841. Il Crist, basandosi sulla scelta del Boca, sull'Etude di Edmond-René Labande (1940) e sulle proprie ricerche, segue il ms. più antico (A), segnalando le differenze tra i due testimoni e adottando con prudenza le lezioni dell'altro ms. (B), soprattutto quando esso permette di colmare le lacune di $\mathrm{A}$, e pubblicando in appendice quella parte di $\mathrm{B}$ che differisce più nettamente da A.

5 È comprensibile che, data la mole dell'opera, si sia cercato di contenere lo spazio dell'Introduzione, e tuttavia si sente la mancanza di qualche indicazione più completa, soprattutto delle posizioni critiche recenti tanto sul ciclo delle Crociate, quanto sulle tarde canzoni di gesta; come pure di un utilizzo più attento del testo per la definizione della personalità dell' autore: indicare in quali altre opere si trovino motivi analoghi, senza pretendere di stabilire rapporti di dipendenza, sembra un atteggiamento di opportuna prudenza, ma si sarebbe potuto tener conto anche, per esempio, delle confuse cognizioni teologiche (il battesimo impartito in nome della Vergine, la confusione tra le persone della Trinità, la confusione tra la Veronica e la Sindone, ecc.), pur segnalate nelle note, in rapporto alla cultura coeva.

6 All'esigenza di brevità si deve probabilmente la descrizione troppo sintetica dei mss., e certo non basta a porvi rimedio il rinvio al Labande e ai lavori dello stesso Cook; ne deriva qualche affermazione arrischiata, come la giustificazione della scelta di A come testo di base:“Comme texte de base, le Ms A s'impose: il est postérieur de peu à la composition du texte", criterio dal punto di vista ecdotico quanto meno discutibile.

7 L'elaborazione di questa edizione ha subito un forte ritardo rispetto alla presentazione (1984) e all'approvazione del Consiglio della Société des Anciens Textes Français (1985), ritardo che ha compromesso l'utilizzo degli strumenti elettronici ed è probabilmente la causa di alcuni aspetti negativi, quali i troppo frequenti refusi sfuggiti ad un'ultima revisione, che avrebbe dovuto essere più accurata, che finiscono per ingenerare qualche interrogativo anche sul testo stesso della chanson. Si rilevano poi imprecisioni di vario tipo: nel paragrafo dell'Introduzione che illustra i criteri seguiti per la grafia del testo, si dice che mortele del v. 14371 è maschile, ma al luogo indicato si trova che mortele è attributo di vie. Per quanto riguarda i nomi propri dallo statuto incerto, per Fauvain si rinvia alla nota al v. 832, nota che però non esiste, mentre nell'indice dei nomi a Fauvain si rinvia al lemma chevaucher del glossario e solo qui si trova l'interpretazione del verso e il rinvio al Tobler-Lommatzsch (III, 1670). Per Anemis si dice che è maiuscolo quando si tratta del diavolo in persona, ma lo troviamo maiuscolo anche al v. 20371 dove leggiamo: “[...] de gramare je suis li Anemis?" Se per gramare si intende "grant maire" (cfr. B), cioè il re di Francia, come peraltro vuole anche il contesto, anemis non può essere che nome comune nell'accezione primitiva; inoltre 
gramare non si trova nel glossario, né nel Dictionnaire de l'ancien français di Greimas, come vorrebbe il criterio di selezione enunciato, secondo il quale si inseriscono nel glossario solo i vocaboli che non si trovano nel citato Dictionnaire, criterio questo pure alquanto problematico.

8 Anche le note si prestano a molte riserve, tanto per l'organizzazione (ora la nota non è segnalata nel testo dall' $n$ in esponente, ora c'è l' $n$ in esponente e manca la nota), quanto per il numero esiguo ( 25 pagine per oltre 25.000 versi) e il contenuto stesso: sono qualche volta superflue: è così importante definire il tipo di bacio della Dame de Pontieu e del sultano (v. 13621)? Il v. 8556: "mais que consaus ne mue, ch'est parlers qui bien siet" che conclude la riflessione degli ospiti di Baudoin, è poi così oscuro?

Talvolta sono poco approfondite e poco persuasive:

- v. 4688: “Au prestre l'esconseille; en bas lui fu disans". La nota rileva che non si trova nei lessici il verbo esconseiller e che, se si separano diversamente le parole leggendo les conseille non si capisce il senso dell'oggetto les. Probabilmente è valida la lezione di B: "se conseille".

11 - v. 13607: "tant le mena la dame de coquet en fablel". Si tratta della Dame de Pontieu che intrattiene il marito nella tour Abel e nel corso del colloquio gli chiede di inviare dei prigionieri cristiani in Francia a cercare il fratello Jean; per la locuzione de coquet en fablel la nota rinvia a Tobler-Lommatzch (II, 514) e spiega: espediente retorico, che significa "ici quelque chose comme leurrer, mener par le bout du nez"; è da prendere in considerazione anche l'interpretazione di Godefroy (s.v.), che cita questo esempio e traduce, forse per analogia con la locuzione du coq à l'ane: "de propos en autre", che bene rappresenta, con connotazione meno negativa, la conversazione volutamente svagata della dama.

12 - v. 16273: "nommet ne doivent estre adès fors que Furcaut". Nell'indice dei nomi Furcaut è interpretato: "«four chaud», semble signifier «idiot» ou d'autres insultes" e si rinvia alla nota, dove si legge: "four chaud, fourche chaude", che non spiega nulla. Non è chiaro chi sia il soggetto plurale di doivent, poiché ha un soggetto singolare anche il verso precedente: "chius qui se fie en prestre trestous ses sens li faut". La locuzione estre en ung chaut four significa: essere "dans le pétrin" (Giuseppe Di Stefano, Dictionnaire des Locutions, s.v. four); forse qui Furcaut può significare: "sciagurato, disgraziato". La lezione di B non è più chiara: "moines ne doivent estre adieux fors que surcaut".

13 - v. 20544:“[...] se par champ en poet li debas estre jus". La nota definisce oscuro il verso, che potrebbe essere interpretato: "la controversia può diventare jus", cioè droit, raison (cfr. Greimas, s.v. jus), se definita par champ, cioè "per mezzo di un un duello".

14 - vv. 21922-23: "Si bien s'i [sont] prouvé li nostre palasin /que Sarrasins reculent a bailles de sapin". La nota spiega que come pronome relativo soggetto plurale; si tratta più correttamente della congiunzione correlativa a $\mathrm{Si}$.

15 Spiace di dover segnalare che il Cook cita nella bibliografia il Dictionnaire di Di Stefano, ma purtroppo non lo utilizza; la consultazione del $D L$ gli avrebbe evitato di affermare, a proposito dei vv.1105-6 ('E, Diex, qu'est che d'argent? Chius le sot bien nommer,/qui argent l'appella: les gens fait embraser"), che si tratta di un'etimologia popolare la quale "semble paraître uniquement dans notre texte", quando il DL ne offre numerosi esempi (s. v. argent); e parimenti gli avrebbe evitato di spiegare "Au four avés esté" (v. 18636) come "vous avez perdu votre temps", quando invece au four significa: "là où l'on sait les nouvelles et surtout les potins" (DL, s.v. four). 

Q apprezzabile la registrazione di un largo contesto, che permette di cogliere i modi d'uso dell'autore, dall'altro la segnalazione di più parole chiave rende complicata la loro classificazione e induce l'autore a ripetere tutto il brano per ognuna di esse, ampliando oltre misura lo spazio dedicato a questa parte. Inoltre, la traduzione in francese moderno di alcune di tali parole non pare necessaria per il lettore di un testo come questo, lettore nel quale genera se mai qualche sconcerto trovare, ad esempio, eür classificato sotto bonheur; caperon sotto chapeau, gentis sotto noble, s'abaubir sotto s'effrayer, engiens sotto intelligence, tresque sotto dance, e per contro danser sotto caroler. Forse, l'ordine alfabetico dei proverbi adottato dal Morawski può apparire elementare, ma è certo di più agevole riferimento, come mostra anche l'uso che ne fa Elisabeth Schulze-Busacker, ignorata, ahimé, nella bibliografia.

Non mi soffermerò sul glossario, terreno minato, e qui in partenza, cioè dal criterio $\mathrm{d}$ selezione dei vocaboli, restrittivo e non sempre osservato, come ho già avuto occasione di notare.

Chi ha qualche esperienza di editore di testi in genere, e particolarmente di testi in antico francese, sa quanto tempo, quanto impegno, quanta fatica esiga tale lavoro, e perciò quanto ingenerosa possa sembrare la critica che si appunta su delle minuzie, su aspetti apparentemente marginali. È però dovere, assai poco gratificante dovere, del recensore ricordare che non esistono in filologia particolari trascurabili e avvertire i lettori dei limiti di affidabilità di un testo: l'edizione Crist del Baudoin de Sebourg è ricca di notizie utili, ed è più accessibile dell'edizione Boca, ma va utilizzata con cautela per quanto riguarda il testo e la sua interpretazione. 